\title{
Plasticity of the Worker Bumblebee Brain in Relation to Age and Rearing Environment
}

\author{
Beryl M. Jones ${ }^{\mathrm{a}}$ Anne S. Leonard ${ }^{c}$ Daniel R. Papaj ${ }^{\mathrm{a}}$ Wulfila Gronenberg $^{\mathrm{b}}$ \\ Departments of ${ }^{\mathrm{a} E c o l o g y}$ and Evolutionary Biology and ${ }^{\mathrm{b}}$ Neuroscience, University of Arizona, Tucson, Ariz., and \\ 'Department of Biology, University of Nevada, Reno, Nev., USA
}

\section{Key Words}

Neuronal plasticity $\cdot$ Bombus $\cdot$ Sensory environment .

Mushroom bodies . Multimodal interactions

\begin{abstract}
The environment experienced during development can dramatically affect the brain, with possible implications for sensory processing, learning, and memory. Although the effects of single sensory modalities on brain development have been repeatedly explored, the additive or interactive effects of multiple modalities have been less thoroughly investigated. We asked how experience with multisensory stimuli affected brain development in the bumblebee Bombus impatiens. First, to establish the timeline of brain development during early adulthood, we estimated regional brain volumes across a range of ages. We discovered significant agerelated volume changes in nearly every region of the brain. Next, to determine whether these changes were dependent upon certain environmental stimuli, we manipulated the visual and olfactory stimuli available to newly emerged bumblebee workers in a factorial manner. Newly emerged bumblebees were maintained in the presence or absence of supplemental visual and/or olfactory stimuli for 7 days, after which the volumes of several brain regions were estimated. We found that the volumes of the mushroom body lobes and
\end{abstract}

calyces were larger in the absence of visual stimuli. Additionally, visual deprivation was associated with the expression of larger antennal lobes, the primary olfactory processing regions of the brain. In contrast, exposure to plant-derived olfactory stimuli did not have a significant effect on brain region volumes. This study is the first to explore the separate and interactive effects of visual and olfactory stimuli on bee brain development. Assessing the timing and sensitivity of brain development is a first step toward understanding how different rearing environments differentially affect regional brain volumes in this species. Our findings suggest that environmental factors experienced during the first week of adulthood can modify bumblebee brain development in many subtle ways.

(c) 2013 S. Karger AG, Basel

\section{Introduction}

Early environmental exposure can have major effects on brain development. For example, classic research in mammalian systems has shown that visual experience in early life is necessary for the proper development and functionality of visual processing systems [Wiesel and Hubel, 1963; Movshon and van Sluyters, 1981; Hooks and Chen, 2007]. More generally, animals reared in enriched

\section{KARGER}

E-Mail karger@karger.com

www.karger.com/bbe
(C) 2013 S. Karger AG, Basel

0006-8977/13/0824-0250\$38.00/0 
environments show increased brain size and weight, larger nuclei in the cortex, and enhanced generation of glial cells, neurite branching, and synapses [e.g. Wiesel, 1981; Kempermann et al., 1998]. In addition to its relevance for animal welfare and conservation [Fleming et al., 1997; Mirescu et al., 2004], understanding how environmental factors shape the development and performance of neural structures has implications for the treatment of human diseases [reviewed in van Praag et al., 2000] and is a central goal of developmental neuropsychology [Gluckman et al., 2005; Lupien et al., 2009].

One of the most intensely studied invertebrate models of neuronal plasticity is the honeybee (Apis mellifera), a social insect which shows age polyethism with respect to worker tasks. Over the course of 2-3 weeks, young honeybees transition from a nurse bee in the dark hive to a forager who must integrate and remember spatial, visual, and olfactory cues associated with foraging [reviewed by Free, 1965; Wilson, 1971; Michener, 1974]. Dramatic structural and organizational neural changes accompany this behavioral shift. Most notably, the volume of the mushroom bodies (MB), involved in multimodal information processing, learning, and memory, expands during the first week after emergence [reviewed by Heisenberg, 2003; Fahrbach, 2006]. Although not as extensive as the expansion associated with foraging, $\mathrm{MB}$ expansion occurs in early life even among honeybees deprived of all light and social interaction [Fahrbach et al., 1998].

In addition to this experience-independent, developmentally preprogrammed MB expansion, honeybee workers also show extensive experience-dependent growth of the $\mathrm{MB}$, as the major input region of the $\mathrm{MB}$ (the calyx) expands greatly with sensory experience and foraging [Withers et al., 1993, 1995; Farris et al., 2001]. Subtle differences in social context can also dramatically affect the honeybee $\mathrm{MB}$, as shown by significant increases in the volume of $\mathrm{MB}$ in honeybees reared with a single dead conspecific compared to those reared alone [Maleszka et al., 2009]. The MB calyces receive and process input from olfactory and other sensory regions of the brain [Mobbs, 1982; Strausfeld et al., 1998; Gronenberg, 2001], and ablation of the MB or mutations in MB-expressed genes in fruit flies result in a number of learning deficits and shortened memory retention [Davis, 1993; de Belle and Heisenberg, 1994; Heisenberg, 2003; Strausfeld, 2012].

Other hymenopterans also exhibit plasticity in the MB, including the ants Camponotus floridanus [Gronenberg et al., 1996], Pheidole dentata [Seid et al., 2005], and Cataglyphis bicolor [Kühn-Bühlmann and Wehner, 2006], as well as paper wasps [Molina and O'Donnell, 2007, 2008; O'Donnell et al., 2007] and the solitary bee Osmia lignaria [Withers et al., 2008]. Similar to what is known from honeybees, the paper wasp Mischocyttarus mastigophorus brain exhibits plasticity associated with age and experience, as well as dominance among nest mates, suggesting that similar brain developmental patterns may exist across the social insects [O'Donnell et al., 2007; Molina and O'Donnell, 2008]. In addition to the MB, the antennal lobes and medulla of the insect brain also show plasticity. The medulla, which receives visual input from the eyes and is involved in primary visual processing, is affected by visual experience in Drosophila melanogaster [Heisenberg et al., 1995; Barth et al., 1997]. In honeybees, the antennal lobe, which receives olfactory information from the antennae, exhibits activity-dependent volume increases in particular antennal glomeruli, accompanied by improvements in associative learning performance [Winnington et al., 1996; Sigg et al., 1997], and classical conditioning leads to changes in the neural representations of odors in olfactory glomeruli [Rath et al., 2011].

Although previous research has established basic information about the extent of brain plasticity among insects, the role of particular sensory stimuli in brain development is greatly understudied, perhaps due in part to the difficulty of controlling the sensory environment of many species in the laboratory. Bumblebees (Bombus) present an appealing system in this respect, as colonies can easily be maintained in a controlled lab environment, and exhibit foraging behavior in the lab that is similar in key aspects to foraging in nature [Bombus terrestris; Raine and Chittka, 2007, 2008]. Additionally, the increasing use of bumblebees in learning studies [e.g. Kulahci et al., 2008; Riveros and Gronenberg, 2009, 2012; Leonard et al., 2011] makes them an attractive candidate for research on brain development, in which learning performance can potentially be connected to underlying neuronal plasticity.

Finally, bumblebees present an interesting contrast to the well-studied honeybee in terms of understanding the interplay between neural plasticity and colony organization. Honeybees have a well-defined age-based division of labor and do not typically begin foraging until approximately 3 weeks after eclosion [Winston, 1987; Robinson, 1992]. In contrast, bumblebee colonies lack an age-based division of labor. Instead, individuals show patterns of weak specialization on different tasks [Jandt and Dornhaus, 2011]. Thus, unlike in honeybees, some bumblebee workers may leave the colony to forage as soon as 2 days after eclosion in laboratory colonies [Riveros and Gronen- 
berg, 2009]. This propensity to forage early is affected in part by body size, which is highly variable within a colony and contributes to task specialization, with larger bees being more likely to forage than smaller bees [Brian, 1952; Free, 1955]. Given that newly emerged bumblebees may engage in a variety of different nursing and foraging tasks, do their brains show patterns of experience-dependent and independent change similar to those seen in honeybees? Describing the factors affecting brain growth in bumblebees is a first step toward understanding the connections between life history traits and neuronal plasticity among social insects.

We assessed brain morphometric changes associated with age and experience in lab-reared colonies of the common eastern bumblebee Bombus impatiens. We first established the timeline of brain growth and composition and then asked whether visual and/or olfactory inputs affect this development independently of age and the social environment of the colony. In particular, we manipulated foraging-related sensory stimulation (specifically, the presence or absence of plant-derived odors and floral colors) in order to determine whether these sensory inputs are associated with changes in the brain. We then compared the relative volumes of sensory brain regions to assess how they reflect exposure to sensory stimuli during development.

Because bumblebees are capable of foraging shortly after emergence, we hypothesized that their brains might be more developed at emergence and thus exhibit less postemergence expansion of sensory regions compared with honeybees. Alternatively, early foraging experience might lead to rapid expansion of particular brain regions, reflecting exposure to visual stimuli, olfactory stimuli, or their combined (additive or interactive) effects. Additionally, because bees have a rich olfactory environment within the colony throughout adulthood but relatively less visual stimuli within the dark hive, we hypothesized that visual stimuli might be more powerful modulators of brain development than the presence or absence of floral odors.

\section{Materials and Methods}

\section{Animals and Rearing Conditions}

Colonies $(\mathrm{n}=5)$ of $B$. impatiens were obtained from Koppert Biological Systems (Howell, Mich., USA). Bees were provided with pollen and Koppert's BeeHappy ${ }^{\circledR}$ solution ad libitum. Colonies were housed in plastic boxes $(\mathrm{L} \times \mathrm{W} \times \mathrm{H}: 22 \times 24 \times 12 \mathrm{~cm})$ on a bed of Feline Pine ${ }^{\circledR}$ cat litter to reduce moisture and odor buildup, and fed from a cotton wick feeder within a small Plexiglas foraging box attached via plastic tubing. Only workers were used for experiments and all workers were marked using numbered tags (E.H. Thorne Ltd., Wragby, UK) glued to their thorax.

\section{Assessment of Age-Related Changes in Regional Brain} Volumes

Bumblebees from 2 colonies were marked as callows $[<12 \mathrm{~h}$ postemergence, recognizable by features of their external appearance; Goulson, 2003] and returned to their colony without further manipulation. Since bees were marked as callows, it was impossible to predict the extent to which each individual would later perform different tasks. Although foragers are on average larger than nest bees [B. terrestris, Goulson et al., 2002] and body size is moderately correlated with an increased probability to forage at an earlier age, $B$. impatiens workers show only weak long-term task specialization [Jandt and Dornhaus, 2011]. In a study of B. bifarius nearcticus with 20 - to 90 -min observations just twice daily up to 5 times a week, at least $88 \%$ of workers were observed to forage at some point in their life [O'Donnell et al., 2000]. Thus, marked individuals were randomly assigned (independently of body size) to one of the following time points to be collected for dissection: 0 (callow), 1, 2, 3, 7, 10, 12, 14, or 21 days after emergence. Colonies were maintained in the lab and had exposure to fluorescent light (Sylvania Cool White $34 \mathrm{~W}, 60 \mathrm{~Hz}, \sim 480 \mathrm{lux}$ ) for $12 \mathrm{~h}$ each day. These colonies were connected to a foraging arena $(\mathrm{L} \times \mathrm{W} \times \mathrm{H}$ : $30.5 \times 30.5 \times 30.5 \mathrm{~cm}$ ) equipped with purple and white artificial flowers, BeeHappy solution in a cotton wick feeder, and 2 floral scents (geraniol and sweet orange, $2 \mu \mathrm{l}$ undiluted essential oil on cotton swabs in arena). Many marked bees were observed foraging in this arena.

\section{Effects of Exposure to Sensory Stimuli on Regional Brain} Volumes

Bees were taken from colonies $(\mathrm{n}=3$ ) maintained in total darkness, ensuring that newly emerged (callow) bees had no prior exposure to light. Workers were marked as callows and assigned to Plexiglas experimental chambers $(\mathrm{L} \times \mathrm{W} \times \mathrm{H}: 30.5 \times 20.3 \times 3.8 \mathrm{~cm})$. While bees could walk freely in these shallow boxes, they could not fly. In order to maintain a standard number of bees present per chamber, supplementary workers (not used in the analysis) were added or removed such that there were always 12 bees per box. Bees received no pollen to minimize ovarian development [Duchateau and Velthuis, 1989] and received $40 \%$ sucrose (weight/weight) solution ad libitum. Dissection of subjects' ovaries later revealed no significant development, suggesting that a shift to reproduction did not take place during the 7 days of isolation from the queen.

Bees in a given chamber were assigned to one of four treatments: (1) visual deprivation and olfactory impoverishment, (2) visual enrichment and olfactory impoverishment, (3) visual deprivation and olfactory enrichment, or (4) visual and olfactory enrichment. Bees were randomly assigned to a treatment, and the intertegular span of each bee was measured as a proxy for body size [Cane, 1987]. The intertegular span was distributed continuously. The mean intertegular spans $( \pm \mathrm{SD})$ for the treatment groups were: $4.50 \pm 0.64,4.46 \pm 0.41,4.26 \pm 0.36$, and $4.55 \pm 0.53$ for Vis-/ Olf-, Vis-/Olf+, Vis+/Olf-, and Vis+/Olf+, respectively. No pair of treatments differed significantly in intertegular span ( $\mathrm{t}$ tests, all $\mathrm{p}>0.05)$.

Bees in a visual deprivation treatment were placed in black opaque boxes and kept under light-proof fabric at all times. Bees in 
a visual-enrichment treatment were placed in clear boxes of identical construction and were exposed to standard fluorescent room lighting (Sylvania Cool White $34 \mathrm{~W}, 60 \mathrm{~Hz}, \sim 480 \mathrm{lux}$ ) and LEDs (peak wavelengths of 395, 420, 476, and $561 \mathrm{~nm}$ and one 'white', broad-spectrum-emitting LED; see online suppl. fig. S1 and S2 for emission spectra [Skorupski and Chittka, 2010]; see www. karger.com/doi/10.1159/000355845 for all online supplementary material) for $10 \mathrm{~h}$ each day. Two LEDs of each type (10 LEDs total) were randomly arranged on the upper surface of each box. The colors of the LEDs were selected to represent colors that foraging bees might encounter when visiting flowers.

While bees were deprived absolutely of visual stimuli, this could not be done for olfactory stimuli because bees themselves produce odors. Instead, chambers were either enriched with additional, plant-derived, odors or not. Bees in an olfactory-enrichment treatment were sequentially exposed to one essential oil per day for 7 days (lavender, grapefruit, peppermint, geranium, cinnamon, ylang-ylang, or jasmine; obtained from oilshop.com) for $10 \mathrm{~h}$ each day. Three microliters of the respective oils were pipetted onto filter paper placed within the box in a mesh-covered dish, ensuring that bees could not make direct physical contact with the oils. Boxes were sealed and a weak airflow was generated by drawing carbon-filtered room air through the box via a vacuum line. In the unsupplemented odor treatment, bees were kept in identical boxes subject to the same weak airflow but without the addition of the plant-derived odors. After 7 days in the chamber, bees were collected for dissection. A sampling point of 7 days was selected based on 2 factors: (1) the potential for some bumblebee workers to forage within 2 days of emergence [Riveros and Gronenberg, 2009] may indicate that their brains are shaped by experience early during the first week of emergence, and (2) the age-based brain development observed in this study shows a plateau in regional brain volume growth around 7 days postemergence (see Results).

\section{Tissue Preparation}

After collection, we immediately decapitated subjects and removed their mandibles and part of each eye to allow fixative to penetrate. For logistical reasons and in order to increase the sample size, brains were batch processed according to age and treatment group. Therefore, it was not possible to compare absolute brain sizes to the size of individual bees. Brains were fixed within the head capsule in $4 \%$ formaldehyde in cacodylate buffer ( $\mathrm{pH} 6.8$ ) overnight on a rotator. After fixation, brains were rinsed with and stored in cacodylate buffer at $4^{\circ} \mathrm{C}$ until dissection. Brains were dissected from the head capsule and then stained in the dark using $1 \%$ aqueous osmium tetroxide for $2 \mathrm{~h}$ on ice, followed by an additional $30 \mathrm{~min}$ at room temperature. After rinsing with distilled water, brains were dehydrated using 50\% ethanol, acidified 2,2-dimethoxypropane [Thorpe and Harvey, 1979], and acetone for $10 \mathrm{~min}$ each. Next, brains were plastic-embedded in Spurr's low-viscosity medium (RT 14300; Electron Microscopy Sciences). Blocks were polymerized at $65^{\circ} \mathrm{C}$ for $12 \mathrm{~h}$, sectioned on a sliding microtome at 10 or $15 \mu \mathrm{m}$ (depending on the extent of the staining and the hardness of the brain; some brains were more brittle and had to be sectioned at $10 \mu \mathrm{m}$ to avoid damage), mounted, and coverslipped.

\section{Brain Volume Estimations}

A camera lucida attachment to a light microscope was used to trace the outlines of one hemisphere of each brain as well as individual brain regions within that hemisphere section by section (ap- prox. $\times 100$ magnification on paper). Specifically, the following brain neuropils were identified by their dark staining properties (fig. 1) and their boundaries drawn: optic lobes (medulla and lobula, which are involved in primary processing of visual information), antennal lobe (receiving input from the antenna for primary processing of olfactory information), central body [suggested to function in the coordination of motor control; Strauss, 2002], and the MB (involved in learning, memory, and multisensory integration [reviewed in Heisenberg, 1998]; lobes and calyces were drawn separately). The volume of cell bodies was also recorded and the remaining neuropil not designated as a region mentioned above is referred to as 'neuropil (rest)' and comprises the remaining protocerebral neuropil, the deutocerebrum except for the antennal lobes, the tritocerebrum, and the neuropilar region of the subesophageal ganglion. Drawings were made by an observer blind to treatment, and the boundaries of the brain regions were determined based on visual inspection of changes in the texture and staining of neuropil in each region (a representative tracing can be seen in fig. 1). Drawings of the brain hemispheres were digitized on a flatbed scanner at $300 \mathrm{dpi}$, and the areas of the respective brain components were estimated using the pixel-counting routine in Adobe Photoshop v7.0 (Adobe Systems, San Jose, Calif., USA). Volumes were determined by multiplying the area of each region by the section thickness. To estimate volumes, every other section (15- $\mu \mathrm{m}$ section thickness) or every third section $(10-\mu \mathrm{m}$ section thickness) was drawn and measured. This method leads to an increase in error of measurement of less than 5\% compared to measuring each section [Mares et al., 2005]. Bumblebees are recognized for a large variation in the body size of workers [Goulson et al., 2002], so to compare across individuals the volumes of different brain regions must be corrected for body size. Previous studies have shown that brain size in bumblebees correlates with head width and body mass [Mares et al., 2005], and thus brain regional volumes were normalized relative to total brain volume (including cell bodies) to account for the variation in size among individuals. In addition to normalizing relative to total brain volume, the volume of brain regions relative to the following brain regions was also measured: (1) total brain - region of interest, (2) neuropil (rest), (3) neuropil (rest) and cell bodies, and (4) all neuropil (total brain without cell bodies) (online suppl. table S1).

\section{Statistical Analyses}

All statistical analyses were performed in SigmaPlot (Systat Software, San Jose, Calif., USA). Data were confirmed to have normal distributions and equal variance for ANOVA. For the age experiment, a post hoc Tukey HSD test was applied to analyze significant changes in brain regions associated with age. To determine the effects of sensory experience on brain size, a two-way ANOVA was used with visual and olfactory stimuli as factors.

\section{Results}

\section{Age-Related Changes in Regional Brain Volumes}

Significant relative volume increases were observed in every region of the brain with the exception of the cell bodies and central body during the first 21 days of adult life. While the central body did not significantly change in rel- 
Fig. 1. Montage of 3 frontal sections through a B. impatiens brain showing major brain components (top) and corresponding outline highlighting regions of interest (bottom). The volumes of the following regions were measured: medulla $(\mathrm{Me})$, lobula (Lo), antennal lobe (AL), MB lobes (vertical lobe, VL; medial lobe, ML, and peduncle, $\mathrm{Pe}$ ), $\mathrm{MB}$ calyces (lateral calyx, $\mathrm{lCa}$; medial calyx, mCa), central body (CB), neuropil (rest) (Ne), subesophageal ganglion (SEG), and cell bodies (remaining shaded area). Other structures labeled include the retina $(\mathrm{Re})$ and lamina (La). Modified from Riveros and Gronenberg [2010].



ative size over time ( $\mathrm{p}=0.7544)$, the relative volume associated with cell bodies decreased by $23 \%$ within the first 2 days after emergence, with another significant decrease after 7 days of adult life (fig. 2a). The decrease in relative cell body volume was associated with an increase in the volume of every neuropilar region of the brain, including the optic lobes, antennal lobe, $\mathrm{MB}$, and remaining neuropil.

The components of the optic lobes, the medulla and lobula, both increased in relative volume by $14.8 \%$ within 2 days of emergence (fig. 2c), with peak relative volumes at 14 and 12 days, respectively. A significant increase in the relative volume of the antennal lobe was observed after just 1 day, with an increase of $16 \%$ relative to newly emerged bees (fig. 2b). The increase in relative volume of the antennal lobe then remained nonsignificant until after 7 days, with a peak in relative volume at 12 days postemergence, an additional increase of $12 \%$ relative to 1-day-old bees.
MB calyces and lobes (fig. 2d) show developmental trends similar to those of the optic lobes and antennal lobe. An increase in relative $\mathrm{MB}$ calyx volume occurred over the first 7 days of adult life, with an overall increase in relative volume of $20.3 \%$ after 3 weeks. The MB lobes appeared to expand even more rapidly, and were significantly larger after just 2 days of adulthood, reaching a total increase of $22.7 \%$ at 21 days.

\section{Effects of Sensory Experience on Brain Components}

Bees that were reared under variable sensory environments for 7 days after emergence were assessed for differences in regional brain volumes. There was no significant difference in intertegular span among treatments based on an ANOVA ( $p=0.381)$. We also observed no significant difference in brain size among treatments $(\mathrm{n}=40$, $\mathrm{F}_{3,36}, \mathrm{p}=0.5680$ ). The average brain size was $1.509 \mathrm{~mm}^{3}$, with a standard deviation of $0.203 \mathrm{~mm}^{3}$ (this large varia- 



Fig. 2. Change in the relative volume of brain regions [cell bodies (a), antennal lobes (b), medulla (top) and lobula (bottom) (c), and MB calyces (top) and lobes (bottom) (d)] in bees ranging from 0 to 21 days old. Symbols represent mean values and error bars are \pm 1 standard error from the mean. Different letters denote significant differences between groups ( $\mathrm{p}<0.05, \mathrm{n}=5$ bees per age); ages marked by the same letter are not significantly different (Tukey HSD post hoc analysis following ANOVA). 
Table 1. Brain subregion volume estimates (mean relative volume \pm SD) in sensory treatments

\begin{tabular}{|c|c|c|c|c|c|c|c|c|c|c|}
\hline Vis & Olf & $\mathrm{n}$ & Medulla & Lobula & Antennal lobe & MB calyces & MB lobes & Central body & Neuropil (rest) & Cell bodies \\
\hline- & - & 10 & $0.1862 \pm 0.0122$ & $0.056 \pm 0.0027$ & $0.0385 \pm 0.0050$ & $0.1096 \pm 0.0080$ & $0.0727 \pm 0.0044$ & $0.0031 \pm 0.0003$ & $0.2385 \pm 0.0147$ & $0.2954 \pm 0.0240$ \\
\hline- & + & 10 & $0.1808 \pm 0.0102$ & $0.0533 \pm 0.0046$ & $0.0368 \pm 0.0036$ & $0.1071 \pm 0.0086$ & $0.0703 \pm 0.0052$ & $0.0032 \pm 0.0003$ & $0.2412 \pm 0.0125$ & $0.3074 \pm 0.0284$ \\
\hline+ & - & 10 & $0.1789 \pm 0.0080$ & $0.0545 \pm 0.0021$ & $0.0318 \pm 0.0026$ & $0.0995 \pm 0.0082$ & $0.0683 \pm 0.0033$ & $0.0033 \pm 0.0002$ & $0.2388 \pm 0.0119$ & $0.3251 \pm 0.0221$ \\
\hline+ & + & 10 & $0.1815 \pm 0.0088$ & $0.0535 \pm 0.0020$ & $0.033 \pm 0.0034$ & $0.1039 \pm 0.0062$ & $0.0670 \pm 0.0048$ & $0.0033 \pm 0.0003$ & $0.2350 \pm 0.0149$ & $0.3228 \pm 0.0193$ \\
\hline Visual effect ${ }^{\mathrm{a}}$ & & & 1.097 & 0.480 & $19.418^{* * *}$ & $7.263^{*}$ & $7.367^{*}$ & 0.0747 & 0.467 & $9.068^{* *}$ \\
\hline Olfactory effect ${ }^{\mathrm{a}}$ & & & 0.200 & 3.696 & 0.045 & 0.157 & 1.757 & 2.642 & 0.0188 & 0.416 \\
\hline Visual $\bullet$ olfactory effect ${ }^{\mathrm{a}}$ & & & 1.660 & 0.690 & 1.537 & 1.985 & 0.129 & 1.316 & 0.578 & 0.903 \\
\hline
\end{tabular}

Vis and Olf refer to whether the bee received $(+)$ or did not receive $(-)$ visual stimuli or olfactory enrichment. Bold type values represent significant effects with $* \mathrm{p}<0.05, * * \mathrm{p}<$ $0.01,{ }^{* * *} \mathrm{p}<0.0001$. ${ }^{\text {a }}$ Values correspond to $\mathrm{F}_{3,36}$ statistics from two-way ANOVA with visual, olfactory, and visual•olfactory factors.

tion in brain size corresponds to the pronounced body size variation of individuals within any given bumblebee colony). The smallest brain measured was $0.918 \mathrm{~mm}^{3}$, and the largest brain measured was $1.855 \mathrm{~mm}^{3}$. The largest brain region measured relative to the total brain was the volume occupied by cell bodies (mean $0.235 \pm 0.0333$ $\mathrm{mm}^{3}$ ) and the smallest brain region measured was the central body (mean $4.7 \pm 0.6 \times 10^{-3} \mathrm{~mm}^{3}$ ).

Table 1 summarizes the relative volumes of each region of the brain measured in the four treatment manipulations. Bees deprived of visual stimuli for 7 days had a larger relative volume of the antennal lobe (fig. $3 ; \mathrm{n}=40$, $\left.\mathrm{F}_{3,36}, \mathrm{p}<0.001\right)$ than bees exposed to visual stimuli. This result was highly significant regardless of which measure served as the reference for normalization (online suppl. table S1). Based on the total brain volume as a reference, neither the level of visual stimuli nor that of olfactory stimuli affected the relative volume of either component of the optic lobes, the medulla or lobula (for relative data based on other reference volumes, see online suppl. table S1). Exposure to different sensory environments also had no effect on the relative volume of the central bodies, which did not differ among treatment groups.

Like the antennal lobes, both the calyces and the lobes of the MB were significantly affected by exposure to visual stimuli (table 1; fig. 4; for relative data based on other reference volumes, see online suppl. table S1). Regardless of exposure to additional olfactory stimuli, among bees provided with visual stimuli, the relative volumes of $\mathrm{MB}$ calyces and lobes were significantly smaller (fig. 4; $\mathrm{n}=40, \mathrm{~F}_{3,36}, \mathrm{p}=0.011$ and $\mathrm{p}=0.010$, respectively) than those of bees deprived of visual stimuli. The smaller relative volume observed in the antennal lobes and $\mathrm{MB}$ of bees exposed to visual stimuli was compensated for by a significantly larger relative volume of cell bodies $(n=40$, $\left.\mathrm{F}_{3,36}, \mathrm{p}=0.005\right)$.

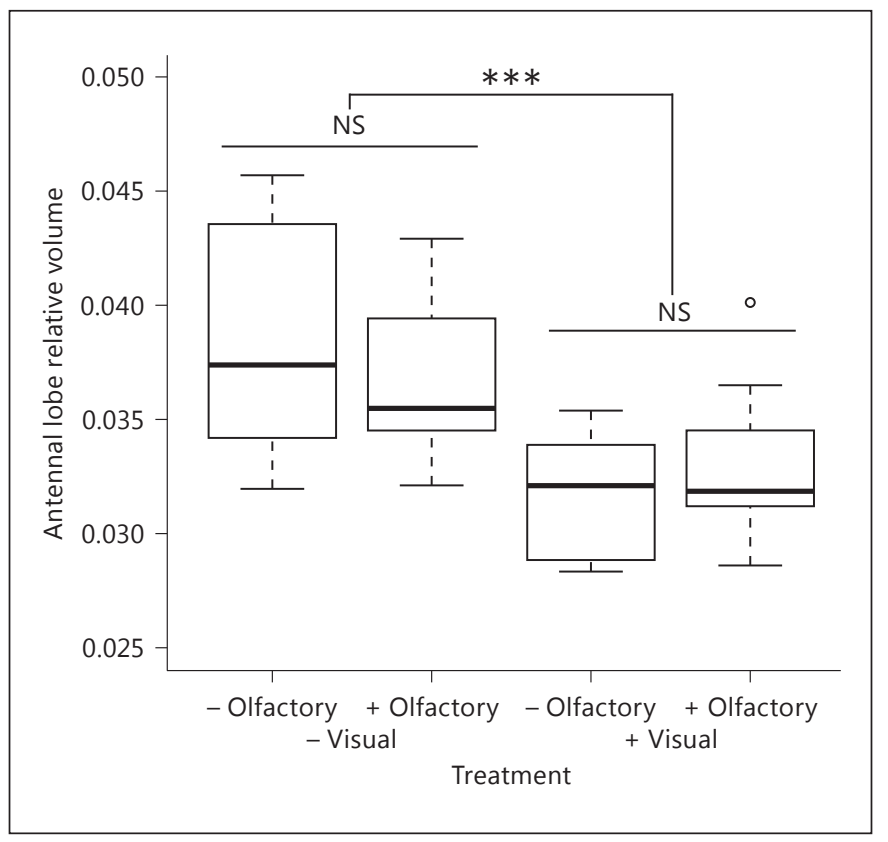

Fig. 3. Effects of visual and olfactory stimuli on the relative volume of antennal lobes. The median of the data is represented by the thick horizontal line, the first and third quartiles are represented by the top and bottom of the rectangle, and the whiskers show the range of the data. Open circles represent statistical outliers in the data set. ${ }^{* * *} \mathrm{p}<0.0001$ obtained from factorial ANOVA, $\mathrm{F}_{3,36}$. $\mathrm{n}=10$ bees per treatment. NS $=$ Not significant.

\section{Discussion}

Our study is the first to explore the effects of both age and experience with multiple sensory modalities on the bumblebee brain and provides a unique point of comparison with the intensively studied honeybee. We discovered significant increases in regional brain volumes that occur soon after emergence, and we determined that 




Fig. 4. Effects of visual and olfactory stimuli on the relative volume of MB calyces (top) and lobes (bottom). The median of the data is represented by the thick horizontal line, the first and third quartiles are represented by the top and bottom of the rectangle, and the whiskers show the range of the data. Circles represent statistical outliers in the data set. ${ }^{*} \mathrm{p}<0.05$ obtained from factorial ANOVA, $\mathrm{F}_{3,36} \mathrm{n}=10$ bees per treatment. NS $=$ Not significant.

within 21 days of adulthood the brain is dramatically restructured in terms of volume allocation. Some of these changes could conceivably occur independently of external stimuli; our study does not permit us to rule out such intrinsic changes. Nevertheless, we also found that bumblebee brain development is sensitive to visual stimuli during the first week of adult life. Since $B$. impatiens workers may forage within 2 days of emergence, we might expect bumblebees to emerge with a brain that is more complete in its development and relatively less plastic than that of the honeybee. However, we found that exposure to stimuli during the first week of life shapes the volumes devoted to both primary sensory processing regions and the $\mathrm{MB}$, sites of sensory consolidation. Interestingly, this plasticity is modality specific: while differences in exposure to visual stimuli led to differences in the relative volume of brain regions, differences in exposure to plantderived olfactory stimuli did not.

Age-Related Volume Increases in the Bumblebee Brain

Consistent with the ability to forage shortly after emergence, bees that remain in a seminatural colony environment showed increases in the relative volume of many sensory processing regions after only a few days of adulthood. The antennal lobes, which are critical for processing chemical stimuli linked to foraging, showed a dramatic relative volume increase $(\sim 37 \%)$ within $10-12$ days. Over $80 \%$ of this increase occurred within the first week (fig. 2b), and one third of the total expansion was observed within the first $72 \mathrm{~h}$. While determining the basis of this early expansion was beyond the scope of this study, it is possible that it relates to the complex chemical environment experienced by newly emerged bees within the nest, in addition to possible early foraging experience.

The medulla and lobula, components of the optic lobes, expand less drastically than the antennal lobes (by 22.2 and $12.2 \%$, respectively, as opposed to $37.4 \%$ ), but nevertheless they increase in relative volume significantly within the first 2 days. While olfaction is clearly important in locating and learning about floral resources, bees at the early stages of foraging may depend more on the visual system than on olfaction for successful navigation. For example, the visual system is also involved in flight control, a basic component of foraging performance that could explain why it appears to be relatively more mature at emergence.

Both measured components of the $\mathrm{MB}$, calyces and lobes, also exhibit significant expansions during the first 3 weeks of adult life. Although there is more variance in these data, much of the significant expansions occur early, within 3 and 7 days, respectively. As the MB have documented roles in sensory integration, learning, and memory [Heisenberg, 2003; Fahrbach, 2006], the expansion of these regions may facilitate foraging, learning, and memory. Alternatively, the observed increase in volume of the MB might be a direct result of early experience. 
The Effect of Visual Experience on Brain Development Having established that bumblebees indeed show changes in brain regions over the course of early adulthood, we next explored to what extent these changes might be shaped by sensory experience. Specifically, we asked whether visual and/or olfactory inputs affect brain development independently of age and the social environment of the colony. Since bumblebees have a rich olfactory environment within the colony throughout adulthood but experience relatively little visual stimuli within the dark hive, we expected that visual stimuli would be more powerful modulators of brain development than floral odors. Consistent with this expectation, we failed to detect significant differences in brain region volumes in relation to the presence or absence of floral odors. In contrast, the presence or absence of visual stimuli had measureable effects on brain region volumes that were independent of the presence or absence of floral odors.

Unlike in D. melanogaster, where the medulla is affected by visual experience [Heisenberg et al., 1995; Barth et al., 1997], neither component of the optic lobes showed differential volume with visual input in this study. However, the absence of volume differences does not rule out more fine-scale changes in the circuitry of the optic lobes. Additionally, the presence or absence of visual stimuli was associated with significant changes in the relative volume of the MB lobes and calyces, suggesting that processing of visual stimuli may be greatly affected by visual experience.

The finding that visual input strongly affects MB volume is consistent with the previously described expansion of MB neuropil in the honeybee, which has been attributed to a particularly dramatic increase in visual input experienced during foraging [Farris et al., 2001]. However, our findings point to a difference in terms of how visual stimuli affect $\mathrm{MB}$ size in honeybees versus bumblebees. Bumblebees that experienced visual input during the first week of adulthood had smaller, not larger, MB volumes compared to bees deprived of visual input. In honeybees, foraging experience leads to an increase in calyx volume, particularly of the visual collar region [Durst et al., 1994], a change that is thought to reflect the increase in visual input associated with leaving the dark hive to forage. In addition to changes associated with the transition to foraging, subtle changes in the social environment of honeybees, such as the presence of a single dead honeybee with a focal individual, lead to significant changes in MB neuropil volume [Maleszka et al., 2009]. If bumblebees are equally sensitive to small changes in the social environment, differences between social interactions in our visually enhanced and deprived treatment groups may have led to some of the effects of visual experience found in our study, which may differ from the effects of visual experience in a natural colony.

While some apparent differences between honeybees and bumblebees in brain development may reflect genuine species differences, they may also reflect differences in methodology. Our experiments systematically manipulated the exposure of sequestered bees to particular kinds of stimuli in a factorial treatment design, whereas many experiments on honeybees [Fahrbach et al., 1997; Farris et al., 2001] sampled unmanipulated bees of various ages in a normal whole-colony and foraging environment. Our experiments thus had the potential to define more precisely the role of particular sensory modalities, and their interaction, in brain development. However, this precision comes at the expense of depriving sequestered bees of activity associated with flight and wholecolony social interactions.

\section{A Cross-Modal Effect of Visual Experience on Antennal Lobe Development}

In addition to affecting MB volume, we found that visual experience also affected antennal lobe volume. Several studies have demonstrated the plasticity of the antennal lobe in honeybees, linking changes in the volume of specific olfactory glomeruli to experience with odors and age [Winnington et al., 1996; Sigg et al., 1997; Brown et al., 2002]. Surprisingly, we found that antennal lobe volume in bumblebees was particularly sensitive to visual input, with odor experience having no observable effect. These results indicate that a focus on single sensory modalities may overlook important changes in the brain associated with other forms of sensory experience. Our finding that antennal lobes are relatively larger in visually deprived bees suggests that bees may exhibit a form of cross-modal reorganization of brain tissue similar to that documented among vertebrates. For example, when cats are visually deprived, they show a significant increase in the number of auditory-responsive cells in the superior colliculus [Rauschecker and Harris, 1983]. In addition, congenitally deaf cats display superior vision in the peripheral field and decreased movement detection thresholds [Lomber et al., 2010]. Brain reorganization has also been widely documented in humans, especially when sensory experience is limited in early development, as in the case of early-onset blindness [reviewed in Noppeney, 2007]. An analogous effect leading to increased olfactoryprocessing tissue volumes in visually deprived bees could explain our results. However, we cannot exclude the pos- 
sibility that absolute brain volume might also have changed as a consequence of the deprivation treatment. Hence it is possible, although not very likely, that the size of visual neuropil stayed the same while that of olfactoryrelated neuropil increased.

The cross-modal effect of visual experience on olfactory centers may also account for the pattern of development of MB calyces and lobes in our study. We did not distinguish the olfactory lip region from the visual collar region of the calyx; hence we do not know which of these regions is reduced in volume by visual experience. Based on findings in Cataglyphis ants [Stieb et al., 2010], one might assume the visual collar region to be affected: in these ants, the number of visual input terminals is reduced in ants exposed to light compared to dark-reared ones. In that study, however, such pruning was associated with an increase in collar volume, rather than the decrease found in the current study (fig. 4). Pruning of the collar region of the MB has also been documented in the paper wasp Polybia aequatorialis, with transitions in behavioral repertoires involving both loss and subsequent growth of dendritic arbors [Jones et al., 2009]. In bumblebees, visual experience might lead to similar dendritic pruning effects which, in turn, might result in a decrease in calyx volume. Alternatively, it might instead be the calyx's olfactory lip region that is altered by visual input, potentially reflecting diminished input from the antennal lobes, which are even more substantially reduced in size in bees provided with visual input (fig. 3).

Finally, our finding that visual stimuli altered brain development raises questions about the specificity of the cross-modal effect. Our visual treatments manipulated multiple aspects of the visual environment simultaneously (e.g. overall light levels, 'floral' colors, and visual stimuli associated with box mates), and thus we cannot yet identify each of these factor's relative contributions. As bees housed in the dark versus light might have had different frequencies of social interactions, untangling the effects of visual experience and visually mediated social interactions on the development of olfactory regions is an obvious next step.

\section{Olfactory Experience and Brain Development in \\ Bumblebees}

In contrast to the effects of visual input, we found no significant volume changes associated with exposure to plant-derived volatiles. Importantly, this result does not mean that the developing bumblebee brain is not sensitive to olfactory input. Short of removing or inactivating the antennae, it is difficult to completely deprive an insect of all olfactory stimuli. Although air entering the chamber was filtered and no outside odorants were introduced, the 12 bees within the chamber undoubtedly contributed olfactory stimuli to their environment, even in plant-odordeprived treatment groups. It is also possible that the addition of plant-derived odors in olfactory treatments affected the brain in ways other than volume. For instance, it may have increased the synaptic density in some antennal glomeruli, as previous studies have shown that synaptic proliferation in the antennal lobe does not necessarily correlate with volumetric changes [Brown et al., 2002]. Because synaptic density or dendritic outgrowth was not measured in this study, we cannot comment conclusively on these potential changes.

Nevertheless, our data suggest that the effect of additional (i.e. plant-derived) odors experienced during foraging on antennal lobe volume may be negligible. In honeybees, the total volume of olfactory glomeruli is significantly larger in nurses than in foragers [Withers et al., 1993]. Considering the olfactory-rich (and visually impoverished) social environment experienced by a nurse bee in the hive, this result may not be surprising. An additional point of consideration is that the antennal lobe of the honeybee requires up to 2 weeks to become fully active, exhibiting changes in the amplitude of calcium signals during glomerular maturation [Shunpeng et al., 2005]. In bumblebees, we have shown that the antennal lobe increases in relative volume by $31.63 \%$ in just 7 days (fig. 2b), suggesting that the network's underlying olfactory processing may also develop rapidly. This rapid expansion may contribute to the ability of bumblebee foragers to forage and learn floral odors on their first day of adulthood [Riveros and Gronenberg, 2009], in contrast to honeybees which do not forage or learn well during their first days of life [Ray and Ferneyhough, 1999].

\section{Conclusion and Future Prospects}

The large-scale reorganization of the bumblebee brain in early life and the ease with which their sensory exposure can be manipulated in a laboratory setting makes them an attractive candidate for studies of environmentdependent neuronal plasticity. As a starting point, we have established that bumblebees exhibit significant neuropil changes associated with both age and visual experience. Unexpectedly, these effects appear to be cross-modal, with visual experience affecting olfactory processing centers in addition to multisensory brain components. In honeybees, neuropil volume increases are associated with 
developmental transitions in behavioral tasks, such as the shift from nursing to foraging with age [Withers et al., 1993, 1995; Farris et al., 2001]. In bumblebees, there is no similar age-related standard shift in task specialization, but workers show neural changes consistent with both age- and experience-dependent shifts in neuropil allocation during early adult life. What is the functional significance of this plasticity? One possibility may be that the weak task specialization found in bumblebee colonies requires a plastic brain, allowing workers to respond to changing colony requirements via alterations in neuronal circuitry. Indeed, our results raise the question of whether the visually mediated plasticity in olfactory processing centers might yield increased performance among darkdwelling workers engaged in chemically complex in-nest tasks, such as larval feeding. As an alternative, perhaps experience-dependent and independent plasticity are common themes in bee brain development regardless of whether tasks are linked with age. Further research on the functional implications of neuropil volume increases in the bumblebee may elucidate the consequences of the changes discovered in this study, as well as establish relationships between neuronal plasticity and life history traits among social insects.

\section{Acknowledgments}

The authors would like to thank R. Kaczorowski and members of the Papaj and Gronenberg labs for helpful discussions.

This work was supported by the National Science Foundation (grant No. IOS-0724591 to W. Gronenberg) and by the University of Arizona Center for Insect Science through the National Institutes of Health (grant No. 1K12 GM000708).

\section{References}

Barth M, Hirsch HVB, Meinertzhagen IA, Heisenberg M (1997): Experience-dependent developmental plasticity in the optic lobe of Drosophila melanogaster. J Neurosci 17: 1493-1504.

Brian AD (1952): Division of labour and foraging in Bombus agorum Fabricius. J Anim Ecol 21: 223-240.

-Brown SM, Napper RM, Thompson CM, Mercer AR (2002): Stereological analysis reveals striking differences in the structural plasticity of two readily identifiable glomeruli in the antennal lobes of the adult worker honeybee. J Neurosci 22:8514-8522.

Cane JH (1987): Estimation of bee size using intertegular span (Apoidea). J Kansas Entomol Soc 60:145-147.

Davis RL (1993): Mushroom bodies and Drosophila learning. Neuron 11:1-14.

$\checkmark$ de Belle JS, Heisenberg M (1994): Associative odor learning in Drosophila abolished by chemical ablation of mushroom bodies. Science 263:692-695.

Duchateau MJ, Velthuis HHW (1989): Ovarian development and egg laying in workers of Bombus terrestris. Entomol Exp Appl 51:199-213.

Durst C, Eichmüller S, Menzel R (1994): Development and experience lead to increased volume of subcompartments of the honeybee mushroom body. Behav Neural Biol 62:259-263.

Fahrbach SE (2006): Structure of the mushroom bodies of the insect brain. Annu Rev Entomol 51:209-232.

- Fahrbach SE, Giray T, Farris SM, Robinson GE (1997): Expansion of the neuropil of the mushroom bodies in male honey bees is coincident with initiation of flight. Neurosci Lett 236:135-138
Fahrbach SE, Moore D, Capaldi EA, Farris SM, Robinson GE (1998): Experience-expectant plasticity in the mushroom bodies of the honeybee. Learn Mem 5:115-123.

Farris SM, Robinson GE, Fahrbach SE (2001): Experience- and age-related outgrowth of intrinsic neurons in the mushroom bodies of the adult worker honeybee. J Neurosci 21: 6395-6404.

Fleming IA, Lamberg A, Jonsson B (1997): Effects of early experience on the reproductive performance of Atlantic salmon. Behav Ecol 8: 470-480.

Free JB (1955): The division of labour within bumblebee colonies. Insect Soc 2:195-212.

Free JB (1965): The allocation of duties among worker honeybees. Sym Zool S 14:39-59.

Gluckman PD, Hanson MA, Spencer HG, Bateson P (2005): Environmental influences during development and their later consequences for health and disease: implications for the interpretation of empirical studies. Proc Biol Sci 272:671-677.

Goulson D (2003): Bumblebees: Behaviour and Ecology. Oxford, Oxford University Press.

-Goulson D, Peat J, Stout JC, Tucker J, Davill B, Derwent LC, Hughes WHO (2002): Can alloethism in workers of the bumblebee Bombus terrestris be explained in terms of foraging efficiency? Anim Behav 64:123-130.

Gronenberg W (2001): Subdivisions of hymenopteran mushroom body calyces by their afferent supply. J Comp Neurol 435:474-489.

Gronenberg W, Heeren S, Hölldobler B (1996): Age-dependent and task-related morphological changes in the brain and the mushroom bodies of the ant Camponotus floridanus. J Exp Biol 199:2011-2019.
Heisenberg M (1998): What do the mushroom bodies do for the insect brain? An introduction. Learn Mem 5:1-10.

Heisenberg M (2003): Mushroom body memoir: from maps to models. Nat Rev Neurosci 4: 266-275.

-Heisenberg M, Heusipp M, Wanke C (1995): Structural plasticity in the Drosophila brain. J Neurosci 15:1951-1960.

Hooks BM, Chen C (2007): Critical periods in the visual system: changing views for a model of experience-dependent plasticity. Neuron 56: 312-326.

Jandt JM, Dornhaus A (2011): Competition and cooperation: bumblebee spatial organization and division of labor may affect worker reproduction late in life. Behav Ecol Sociobiol 65: 2341-2349.

- Jones TA, Donlan NA, O’Donnell S (2009): Growth and pruning of mushroom body Kenyon cell dendrites during worker behavioral development in the paper wasp, Polybia aequatorialis (Hymenoptera: Vespidae). Neurobiol Learn Mem 92:485-495.

Kempermann G, Kuhn HG, Gage FH (1998): Experience-induced neurogenesis in the senescent dentate gyrus. J Neurosci 18:3206-3212.

Kühn-Bühlmann S, Wehner R (2006): Age-dependent and task-related volume changes in the mushroom bodies of visually guided desert ants, Cataglyphis bicolor. J Neurobiol 66: 511-521.

Kulahci IG, Dornhaus A, Papaj DR (2008): Multimodal signals enhance decision making in foraging bumble-bees. Proc Biol Sci 275:797802. 
Leonard AS, Dornhaus A, Papaj DR (2011): Flowers help bees cope with uncertainty: signal detection and the function of complex floral signals. J Exp Biol 214:113-121.

Lomber SG, Meredith MA, Kral A (2010): Crossmodal plasticity in specific auditory cortices underlies visual compensations in the deaf. Nat Neurosci 13:1421-1429.

- Lupien SJ, McEwen BS, Gunnar MR, Heim C (2009): Effects of stress throughout the lifespan on the brain, behavior and cognition. Nat Rev Neurosci 10:434-445.

-Maleszka J, Barron AB, Helliwell PG, Maleszka R (2009): Effect of age, behaviour and social environment on honey bee brain plasticity. J Comp Physiol A Neuroethol Sens Neural Behav Physiol 195:733-740.

Mares S, Ash L, Gronenberg W (2005): Brain allometry in bumblebee and honey bee workers. Brain Behav Evol 66:50-61.

Michener CD (1974): The Social Behavior of the Bees: A Comparative Study. Cambridge, Harvard University Press.

-Mirescu C, Peters JD, Gould E (2004): Early life experience alters response of adult neurogenesis to stress. Nat Neurosci 7:841-846.

-Mobbs PG (1982): The brain of the honeybee Apis mellifera. 1 . The connections and spatial organization of the mushroom bodies. Philos Trans R Soc Lond B Biol Sci 298:309-354.

-Molina Y, O’Donnell S (2007): Mushroom body volume is related to social aggression and ovary development in the paperwasp Polistes instabilis. Brain Behav Evol 70:137-144.

Molina Y, O’Donnell S (2008): Age, sex, and dominance-related mushroom body plasticity in the paperwasp Mischocyttarus mastigophorus. Dev Neurobiol 68:950-959.

Movshon JA, van Sluyters RC (1981): Visual neural development. Annu Rev Psychol 32:477522.

Noppeney U (2007): The effects of visual deprivation on functional and structural organization of the human brain. Neurosci Biobehav R 31:1169-1180.

O'Donnell S, Donlan N, Jones T (2007): Developmental and dominance-associated differences in mushroom body structure in the paper wasp Mischocyttarus mastigophorus. J Neurobiol 67:39-46.
O’Donnell S, Reichardt M, Foster R (2000): Individual and colony factors in bumble bee division of labor (Bombus bifarius nearcticus Handl; Hymenoptera, Apidae). Insectes Soc 47:164-170.

Raine NE, Chittka L (2007): The adaptive significance of sensory bias in a foraging context: floral colour preferences in the bumble bee Bombus terrestris. PLoS One 2:e556.

Raine NE, Chittka L (2008): The correlation of learning speed and natural foraging success in bumble bees. Proc Biol Sci 275:803-808.

Rath L, Galizia CG, Szyszka P (2011): Multiple memory traces after associative learning in the honey bee antennal lobe. Euro J Neurosci 34:352-360.

Rauschecker JP, Harris LR (1983): Auditory compensation of the effects of visual deprivation in the cat's superior colliculus. Exp Brain Res 50:69-83.

Ray S, Ferneyhough B (1999): Behavioral development and olfactory learning in the honeybee (Apis mellifera). Dev Psychobiol 34:2127.

Riveros AJ, Gronenberg W (2009): Olfactory learning and memory in the bumblebee Bombus occidentalis. Naturwissenschaften 96:851-856

-Riveros AJ, Gronenberg W (2010): Brain allometry and neural plasticity in the bumblebee Bombus occidentalis. Brain Behav Evol 75: 138-148.

-Riveros AJ, Gronenberg W (2012): Decisionmaking and associative color learning in harnessed bumblebees (Bombus impatiens) Anim Cogn 15:1183-93.

Robinson GE (1992): Regulation of division of labor in insect societies. Annu Rev Entomol 37: 637-665.

Seid MA, Harris KM, Traniello JFA (2005): Agerelated changes in the number and structure of synapses in the lip region of the mushroom bodies in the ant Pheidole dentata. J Comp Neurol 488:269-277.

-Shunpeng W, Zhang S, Sato K, Srinivasan MV (2005): Maturation of odor representation in the honeybee antennal lobe. J Insect Physiol 51:1244-1254.

-Sigg D, Thompson CM, Mercer AR (1997): Activity-dependent changes to the brain and behavior of the honey bee, Apis mellifera. J Neurosci 17:7148-7156.
Skorupski P, Chittka L (2010): Photoreceptor spectral sensitivity in the bumblebee, Bombus impatiens (Hymenoptera: Apidae). PLoS One 5:e12049.

-Stieb SM, Muenz TS, Wehner R, Rössler W (2010): Visual experience and age affect synaptic organization in the mushroom bodies of the desert ant Cataglyphis fortis. Dev Neurobiol 70:408-423.

Strausfeld NJ (2012): Arthropod Brains: Evolution, Functional Elegance, and Historical Significance. Cambridge, Harvard University Press.

Strausfeld NJ, Hansen L, Li YS, Gomez RS, Ito K (1998): Evolution, discovery, and interpretations of arthropod mushroom bodies. Learn Mem 5:11-37.

- Strauss R (2002): The central complex and the genetic dissection of locomotor behaviour. Curr Opin Neurobiol 12:633-638.

Thorpe JR, Harvey DMR (1979): Optimization and investigation of the use of 2,2-dimethoxypropane as a dehydration agent for plant tissues in TEM. J Ultrastruct Res 68:186-194.

van Praag H, Kempermann G, Gage FH (2000): Neural consequence of environmental enrichment. Nat Rev Neurosci 1:191-198.

Wiesel TN (1981): Physiology or medicine. Nobel lecture, Boston.

Wiesel TN, Hubel DH (1963): Single-cell responses in striate cortex of kittens deprived of vision in one eye. J Neurophysiol 26:1003-17.

Wilson EO (1971): The Insect Societies. Cambridge, Harvard University Press.

-Winnington AP, Napper RM, Mercer AR (1996): Structural plasticity of identified glomeruli in the antennal lobes of the adult worker honey bee. J Comp Neurol 365:479-490.

Winston ML (1987): The Biology of the Honey Bee. Cambridge, Harvard University Press.

Withers GS, Day NF, Talbot EF, Dobson HEM, Wallace CS (2008): Experience-dependent plasticity in the mushroom bodies of the solitary bee Osmia lignaria (Megachilidae). Dev Neurobiol 68:73-82.

Withers GS, Fahrbach SE, Robinson GE (1993): Selective neuroanatomical plasticity and division of labour in the honeybee. Nature 364: 238-240.

Withers GS, Fahrbach SE, Robinson GE (1995): Effects of experience and juvenile hormone on the organization of the mushroom bodies of honey bees. J Neurobiol 26:130-144. 